\title{
Prevention and optimal management of sarcopenia: a review of combined exercise and nutrition interventions to improve muscle outcomes in older people
}

\author{
This article was published in the following Dove Press journal: \\ Clinical Interventions in Aging \\ II May 2015 \\ Number of times this article has been viewed
}

\author{
Hayley J Denison' \\ Cyrus Cooper ${ }^{1,2}$ \\ Avan Aihie Sayer ${ }^{1,2}$ \\ Sian M Robinson ${ }^{1,2}$ \\ 'MRC Lifecourse Epidemiology \\ Unit, University of Southampton, \\ Southampton General Hospital, \\ Southampton, UK; ${ }^{2}$ National Institute \\ for Health Research Southampton \\ Biomedical Research Centre, \\ University of Southampton and \\ University Hospital Southampton \\ NHS Foundation Trust, \\ Southampton, UK
}

\begin{abstract}
The growing recognition of sarcopenia, the age-related loss of skeletal muscle mass and function, has highlighted the need to understand more about its etiology. Declines in muscle mass and strength are expected aspects of aging, but there is significant variability between individuals in rates of loss. Although some of these differences can be explained by fixed factors, such as sex, much of the remaining variation is unexplained. This has led to increasing interest in the influence of adult lifestyle, particularly in the effects of modifiable factors such as physical activity and diet, and in identifying intervention opportunities both to prevent and manage sarcopenia. A number of trials have examined the separate effects of increased exercise or dietary supplementation on muscle mass and physical performance of older adults, but less is known about the extent to which benefits of exercise training could be enhanced when these interventions are combined. In a comprehensive review of the literature, we consider 17 studies of older adults ( $\geq 65$ years) in which combined nutrition and exercise interventions were used to increase muscle strength and/or mass, and achieve improvements in physical performance. The studies were diverse in terms of the participants included (nutritional status, degree of physical frailty), supplementation strategies (differences in nutrients, doses), exercise training (type, frequency), as well as design (duration, setting). The main message is that enhanced benefits of exercise training, when combined with dietary supplementation, have been shown in some trials - indicating potential for future interventions, but that existing evidence is inconsistent. Further studies are needed, particularly of exercise training combined with dietary strategies that increase intakes of a range of nutrients, as well as bioactive non-nutrients, to provide the evidence on which public health and clinical recommendations can be based.
\end{abstract}

Keywords: sarcopenia, diet, exercise, intervention

\section{Introduction}

The importance of sarcopenia, the age-related loss of skeletal muscle mass and function, is now widely recognized. ${ }^{1}$ The decline in muscle mass between 40 and 80 years is estimated to be between $30 \%$ and $50 \%$, with reported losses in functional capacity amounting to as much as 3\% each year beyond the age of $60 .{ }^{2}$ Sarcopenia is a major clinical problem for older people as it is common (with estimates of prevalence as high as $33 \%$ in community-dwelling older adults $)^{2-4}$ and is clearly associated with poorer health consequences, including obesity, osteoporosis, and type 2 diabetes. $^{5-7}$ It is a major cause of frailty ${ }^{6}$ and, importantly, predicts future mortality in middle-aged as well as older adults. ${ }^{7,8}$ The estimated direct health care cost attributable to sarcopenia in the United States in 2000 was $\$ 18.5$ billion. $^{9}$
Correspondence: Avan Aihie Sayer MRC Lifecourse Epidemiology Unit, University of Southampton, Southampton General Hospital, Tremona Road, Southampton SOI6 6YD, UK

Tel +442380764050

Fax +44238070 4021

Email aas@mrc.soton.ac.uk 
The growing recognition of the significance of sarcopenia has led to standardization in operational definitions. ${ }^{6,10,11}$ An example is the consensus statement from the European Working Group on Sarcopenia in Older People (EWGSOP) that recommends a definition of sarcopenia based on threshold values for lean mass, grip strength, and gait speed. The EWGSOP describes the gradation from pre-sarcopenia (decrease in muscle mass without disturbance in strength or function), to sarcopenia (decreased mass and strength or function), to severe sarcopenia (decreased mass, strength, and function). ${ }^{10}$ The increased awareness of the clinical importance of sarcopenia has also highlighted the need to understand more about its etiology. Declines in muscle mass and strength are expected aspects of aging, alongside a range of age-related changes, for example in vascular, endocrine, and neuronal function, ${ }^{6}$ but there is significant variability between individuals in rates of loss. Some of the variability can be explained by factors that are fixed (such as sex, size, heritability, and early life environment), but much of the remaining variation is unexplained. This has led to increasing interest in the influence of adult lifestyle, particularly in the effects of modifiable factors such as physical activity ${ }^{12}$ and diet $^{13}$ on muscle mass and function in older people, with a view to identifying intervention opportunities both to prevent and manage sarcopenia. ${ }^{14}$

Physical inactivity is clearly linked to losses of muscle mass and strength, suggesting that increasing levels of physical activity should have protective effects. A range of exercise interventions has been tested. Of these, progressive resistance exercise training (that requires participants to exercise against an increasing external load) has been most widely used and shown to have positive effects on muscle strength and physical function. ${ }^{15}$ In comparison, less is known about the role of diet in older age, although there is a growing body of evidence that links poor quality diets, and insufficient intakes of protein, vitamin $\mathrm{D}$, antioxidant nutrients, and $\mathrm{n} 3$ long-chain polyunsaturated fatty acids, to poor physical function. ${ }^{13}$ Low food intakes and monotonous diets are common in older age, increasing opportunity for insufficient nutrient intake. Interventions to achieve improvements in diet among older adults who are at risk, may therefore have potential both to prevent or delay age-related losses of muscle mass and function, as well as being potential management strategies for patients who are already sarcopenic. However, existing evidence from nutrient supplementation studies is mixed. ${ }^{4,13}$

Much of the trial evidence evaluates the separate effects of increased exercise or dietary supplementation on muscle mass and function, and an unanswered question is the extent to which benefits could be enhanced when these interventions are combined. ${ }^{16-18} \mathrm{~A}$ meta-analysis of findings from randomized controlled trials has shown that protein supplementation during an exercise training program increases gains in muscle mass and strength, in younger $(<50$ years) and older ( $\geq 50$ years) adults ${ }^{16}$ - although much less is known about the combined effects of exercise training and supplementation with other dietary components that have been linked to sarcopenia. The separate mechanisms of action of nutrition and exercise provide sound physiological rationale for potential additive or synergistic effects, ${ }^{17}$ but the long-term benefits of exercise training combined with dietary supplementation have not yet been clearly established. ${ }^{4} \mathrm{~A}$ further consideration is whether such effects may differ with increasing age or degree of frailty, as much of the existing trial evidence is from younger, healthy participants. For example, in their meta-analysis of protein supplementation studies, Cermak et al speculate that there could be greater gains resulting from combined exercise training and supplementation at more advanced ages ( $>65$ years). ${ }^{16}$

Understanding the benefits of maintaining sufficient levels of physical activity and diet quality in older age is central to considerations of current and future public health strategies to promote better physical function and health in later life. In this paper we describe a comprehensive literature review in which we evaluate the combined effects of dietary supplementation and exercise training on muscle outcomes (strength, mass, and physical performance) in adults aged 65 years and older. The principal aim was to assess evidence of additional benefits of dietary supplementation when combined with exercise training.

\section{Methods}

We sought to include trials which had included both nutritional supplementation and exercise training, and had examined measures of muscle strength, size, and/or physical performance as outcomes. Studies were included if they had comparison groups that received exercise training as well as exercise training combined with nutritional supplementation. We excluded studies that only measured protein synthesis, muscle fiber hypertrophy or biochemical properties of muscle; we also excluded studies where the nutritional intervention was energy restriction to promote weight loss. We restricted the search to studies where the participants were 65 years and older.

To identify eligible studies, we searched the MEDLINE and EMBASE databases via the OVID system for articles which included both MeSH and free-text terms related to 
trials of nutrition, exercise or physical activity and muscle outcomes. The databases were searched from their start dates to April 2013. We limited our search to articles published in English; we excluded animal studies.

Four thousand eight hundred and eighty seven abstracts were identified through database searching and a further ten were identified from screening the references of known review articles (Figure 1). In total, 4,897 abstracts were screened for eligibility by one reviewer (HJD). Conference abstracts were excluded and we did not attempt to obtain unpublished data. One hundred and thirty three papers were considered to be potentially eligible for inclusion and the full-text of these papers was retrieved for further assessment. Twenty-one papers, describing 17 individual studies were eligible for inclusion. ${ }^{19-39}$ The references of included papers were also screened for additional articles, which did not generate any further studies for inclusion.

Relevant data from the included papers were extracted into a table format. Extracted information included details about the study population, the study design, the intervention implemented, and the outcome measures. The studies were not graded for quality; no attempt at a meta-analysis was made.

\section{Results}

Seventeen studies met the inclusion criteria for the review; details of these studies are shown in Table 1. Of these, six studies ${ }^{19-23,27,34,35}$ were based in Europe, six were from the USA or Canada, ${ }^{24,26,28,29,36,38,39}$ two from South America, ${ }^{32,33,37}$ one from Australia, ${ }^{30}$ and two from Japan..$^{25,31}$ The studies ranged in size from 17 participants ${ }^{24}$ to $217^{34,35}$ and varied in duration between 8 weeks $^{26,36}$ and 18 months. ${ }^{32,33}$ Half of the reviewed studies included two comparison groups (exercise, exercise + nutritional supplement), ${ }^{19,22-24,26,31,36,38,39}$ the remaining studies had a factorial design with four groups (exercise, nutritional supplement, both interventions, neither intervention). ${ }^{20,21,25,27-30,32-35,37}$ Study populations varied considerably, from community-dwelling older adults, ${ }^{19,23,24,26,32,33,37-39}$ to older adults with comorbidities such as frailty, ${ }^{22,34,35}$ chronic obstructive pulmonary disease $(\mathrm{COPD})^{31}$ and sarcopenia ${ }^{25}$ and older adults in residential care ${ }^{20,21,27-29,36}$ or who were hospitalized..$^{30}$

The majority of studies (ten of 17) used resistance training as the exercise intervention. ${ }^{19,22-24,28-30,32,33,37-39}$ The remaining seven studies employed a multicomponent exercise program which involved both resistance training and additional exercises such as aerobic exercises, flexibility,

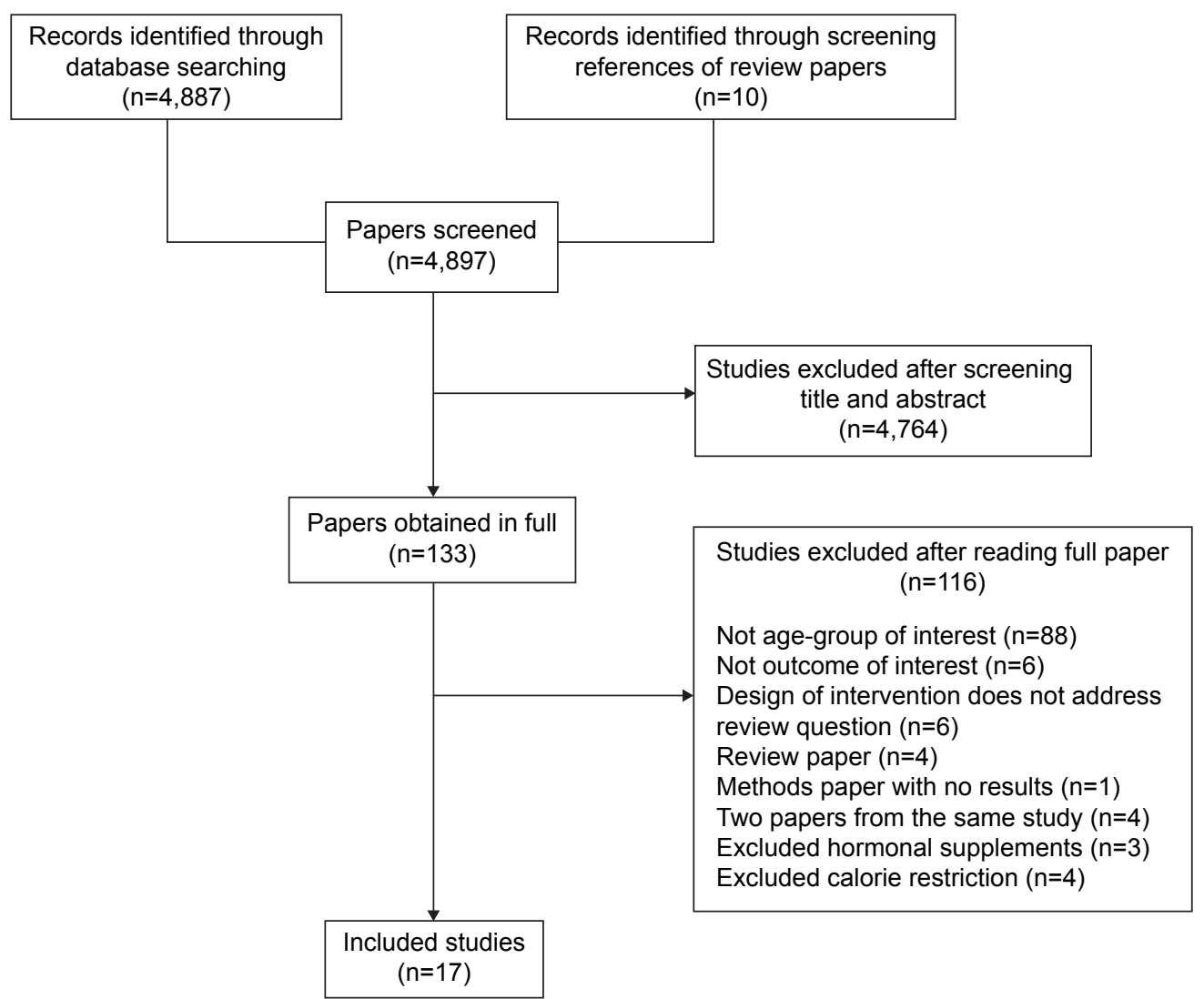

Figure I Flow diagram of literature search. 
and balance..$^{20,21,25-27,31,34-36}$ None of the studies utilized an exercise intervention that was exclusively aerobic exercise. As the exercise training in all studies involved resistance exercise, and to enable studies to be grouped, they were considered together. However, it is important to note that the exact exercises performed, as well as the intensity and duration of the exercise interventions varied widely between studies (Table 1).

The nature of the nutritional supplement used varied between studies in terms of the type of supplement, which additional nutrients were provided, the dose of additional nutrients, the timing of supplementation (at same time as exercise or not), and frequency of consumption. However, whilst acknowledging these differences in study design, overall the 17 studies could be considered within one of four broad groups of nutritional supplements: protein or amino acid supplementation (including $\beta$-hydroxy- $\beta$-methylbutyrate [HMB]) (seven studies), ${ }^{19-26}$ vitamin D supplementation (two studies), ${ }^{36,37}$ multinutrient supplementation (six studies) ${ }^{27-35}$ or creatine supplementation (two studies). ${ }^{38,39}$ The multinutrient supplements used in the interventions were diverse, ranging from a nutritional porridge to fortified food products and milk-based drinks. The micronutrients included therefore differed across these studies. The majority of supplements used in these studies also included protein, but were distinct from the protein/amino acid studies in which multiple micronutrients were not included in the supplements.

Muscle outcomes in these 17 studies included muscle strength in 15 studies, ${ }^{19-26,28-30,32-39}$ a measure of muscle size (cross-sectional area, lean mass, fat free mass) in 15 studies, ${ }^{19-29,31-35,37-39}$ and physical performance in 14 studies. ${ }^{19-22,25,27-39}$ Methods of assessment of each of these outcomes differed across studies. In addition, muscle power (calculated from a power-velocity curve from measurements taken using an Ergo power dynamometer) was assessed in one study, ${ }^{27}$ and muscle endurance in one other. ${ }^{39}$

\section{Types of nutritional intervention Protein/amino acid supplementation}

Four studies ${ }^{19-23}$ used a protein-enriched drink as the nutritional supplement (providing 10-20 g protein); two further studies provided participants with supplements containing essential amino acids (12 g/day, ${ }^{24} 6 \mathrm{~g} /$ day $),{ }^{25}$ one provided a leucine metabolite, HMB (3 g/day). ${ }^{26}$ The seven studies included healthy community-dwelling older adults ${ }^{19,23,24,26 \text {, }}$ as well as sarcopenic ${ }^{25}$ and frail ${ }^{20-22}$ participants. The duration of studies ranged between 8 and 24 weeks; all measured strength and assessed muscle size (as lean body mass/muscle mass or area). All but three studies ${ }^{23,24,26}$ also assessed physical performance.

\section{Muscle strength}

Muscle strength improved with exercise training in all studies. There was no interaction between protein or essential amino acid supplementation with exercise training on muscle strength in six of the studies considered. However, in the study of older Japanese women who were sarcopenic, muscle strength improved only among women who had received the combined intervention of a multicomponent exercise training program and amino acid supplementation. ${ }^{25}$

\section{Muscle size}

The effects of exercise training and supplementation on muscle size varied between studies. In five studies, no interactions were found between exercise training and protein/amino acid supplementation on muscle mass, ${ }^{20,21,23,25}$ cross-sectional area $^{24}$ or lean body mass. ${ }^{19,23}$ However, an increase of $0.8 \mathrm{~kg}$ in lean mass was observed following HMB supplementation of healthy older adults (compared with no change in placebo group; $P=0.08),{ }^{26}$ and interactive effects were observed on lean body mass, among frail older men and women participating in a resistance exercise training program, following provision of a protein-supplemented drink consumed twice per day. ${ }^{22}$

\section{Physical performance}

Physical performance improved with exercise training in all four studies in which it was assessed. ${ }^{19-22,25}$ No additional improvements were found in physical performance in participants who received exercise training and protein or amino acid supplementation.

\section{Multinutrient supplementation}

Six studies provided a multinutrient supplement (nutritional drinks, ${ }^{27-31}$ food products ${ }^{32,33}$ or enriched foods) $)^{34,35}$ for daily consumption. The composition of the supplements differed, but with one exception in which fortified foods were provided to increase micronutrient intake only in the intervention groups, ${ }^{34,35}$ all were complete supplements containing a range of micro- and macronutrients. The nutrients included in the supplements and doses differed across studies. The duration of the interventions ranged from 10 weeks ${ }^{28,29}$ to 18 months, ${ }^{32,33}$ and the majority included older adults who tended to be more vulnerable as they were physically frail, ${ }^{27-29,34,35}$ nutritionally "at risk" 30 and/or had other comorbidities; ${ }^{31}$ one study evaluated the effects of a nutritional supplement provided as part 


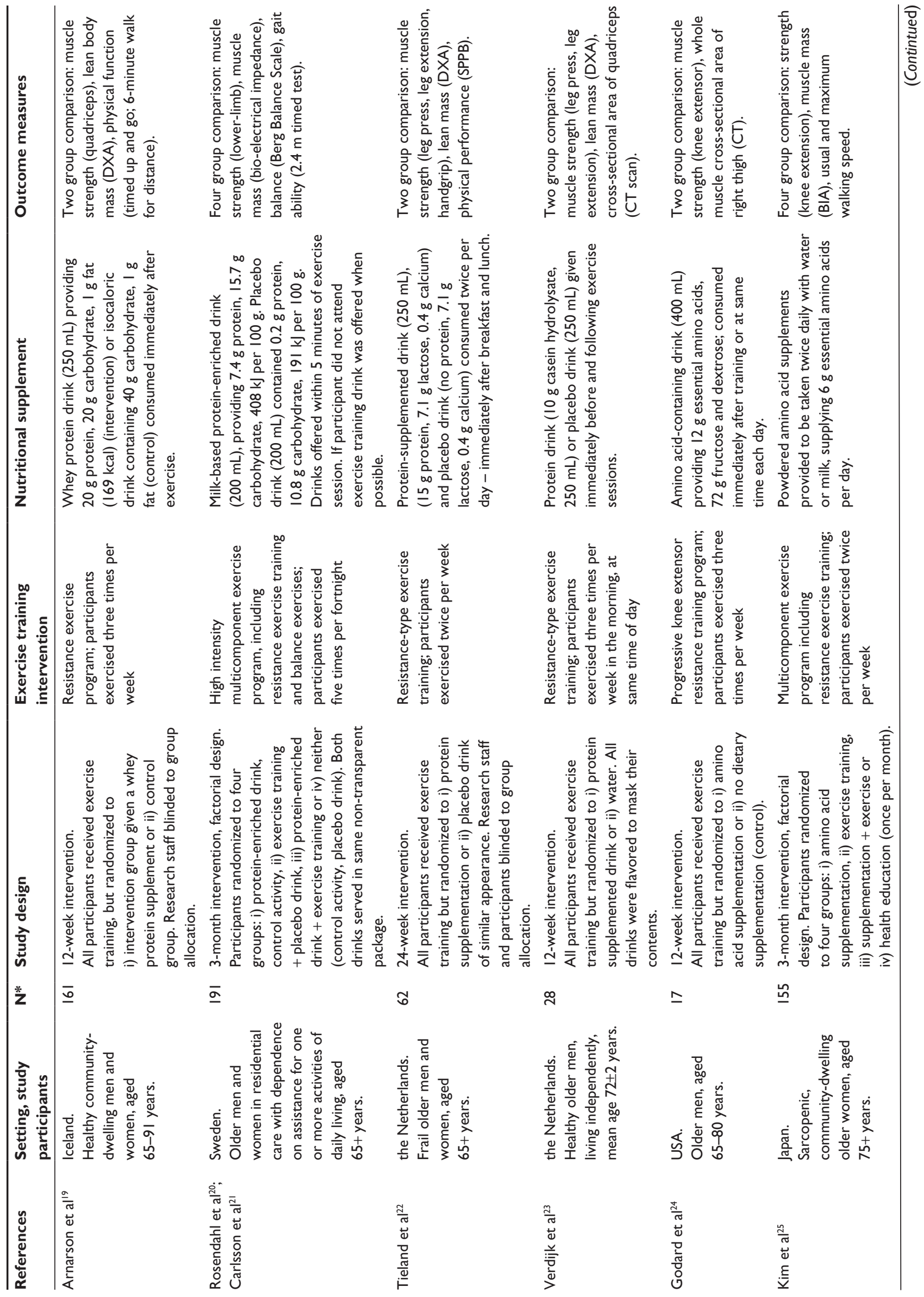




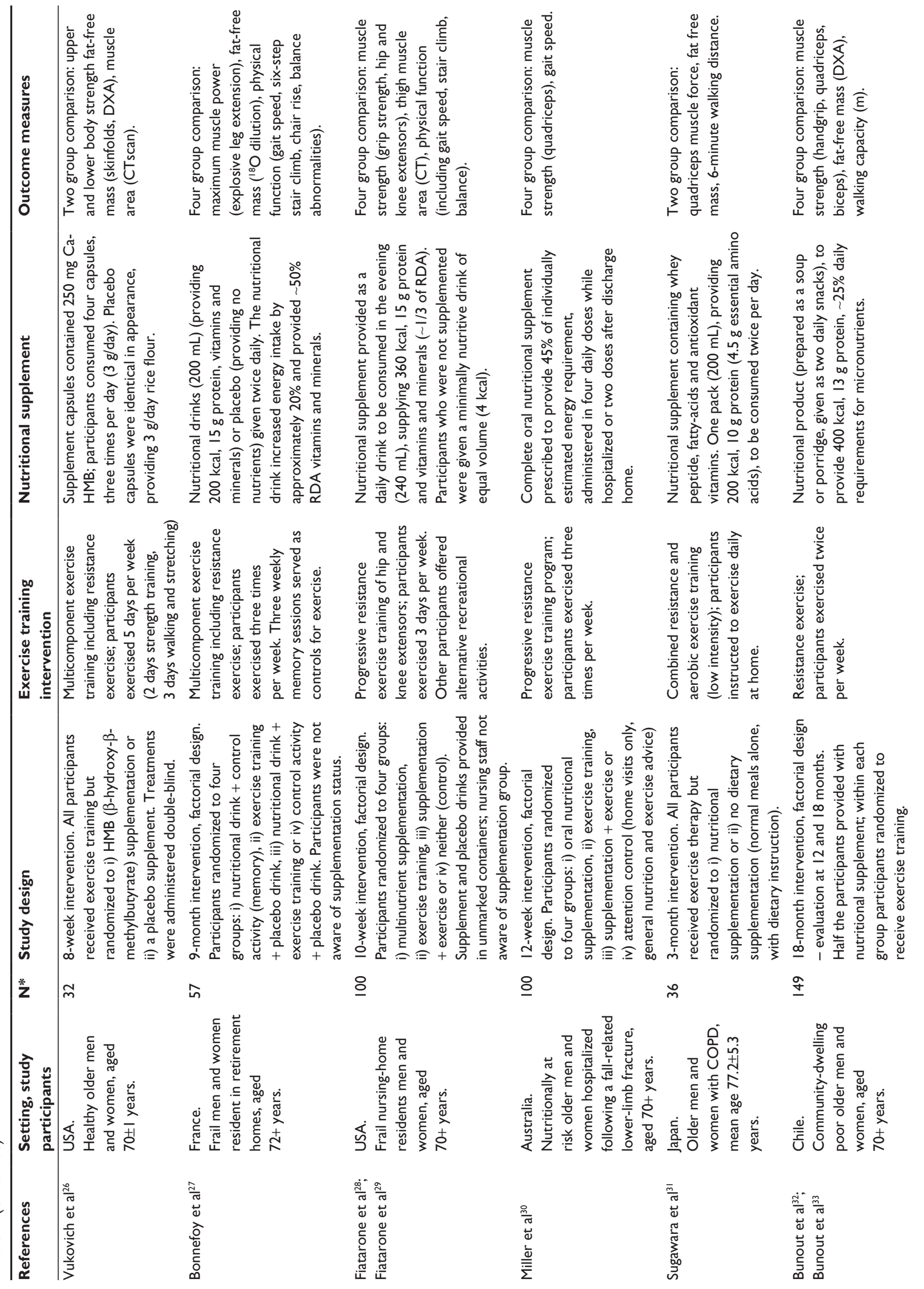



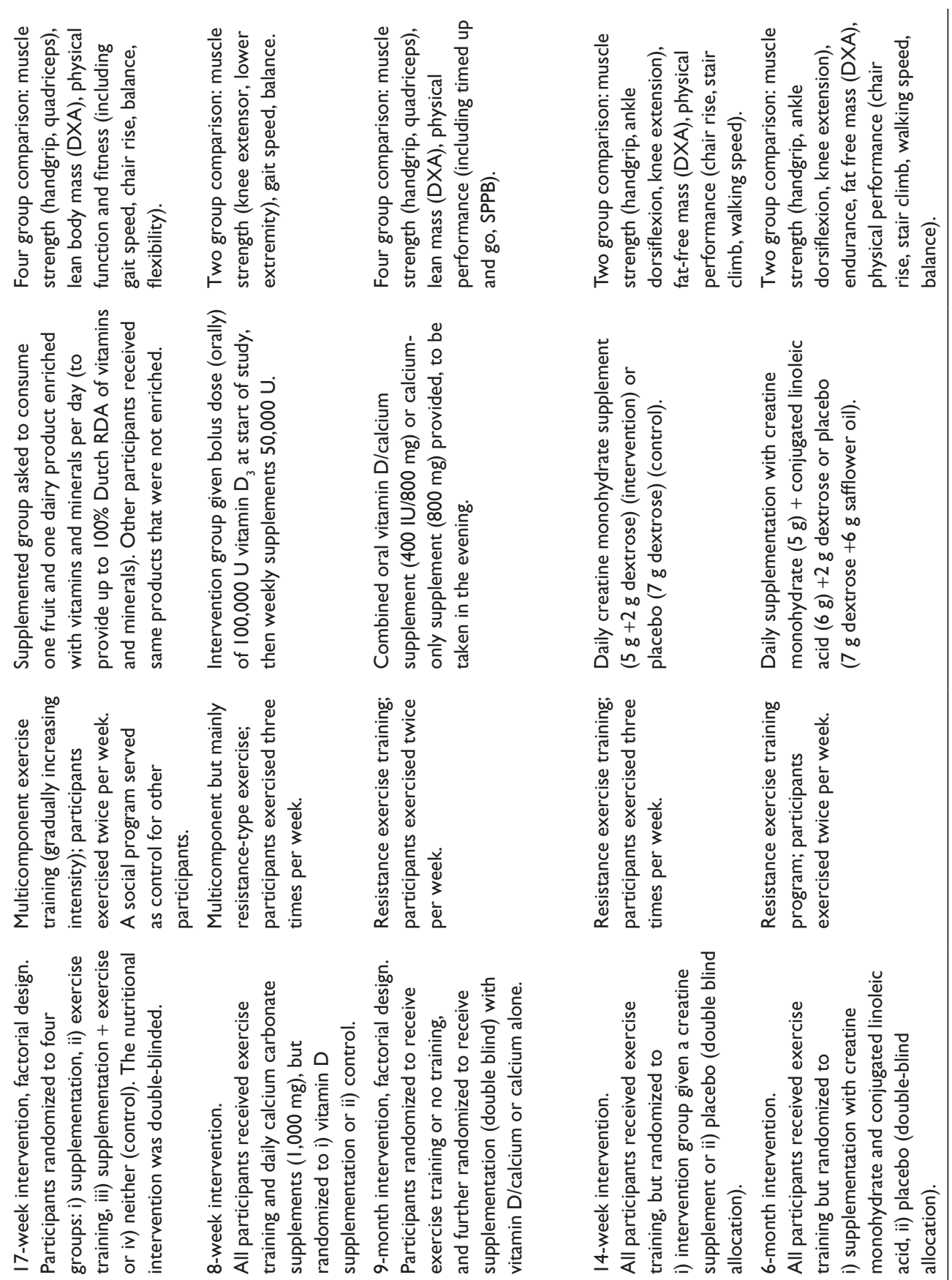

$\stackrel{\bar{N}}{ }$

产

ஃ

ㅇ

㝏

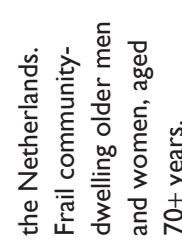

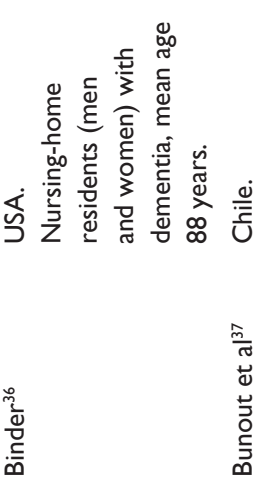

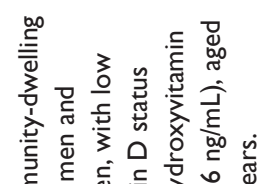

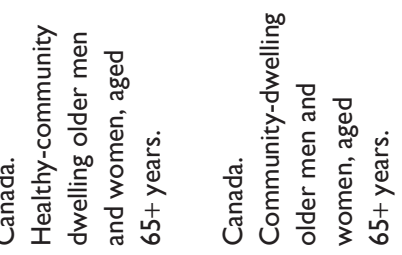
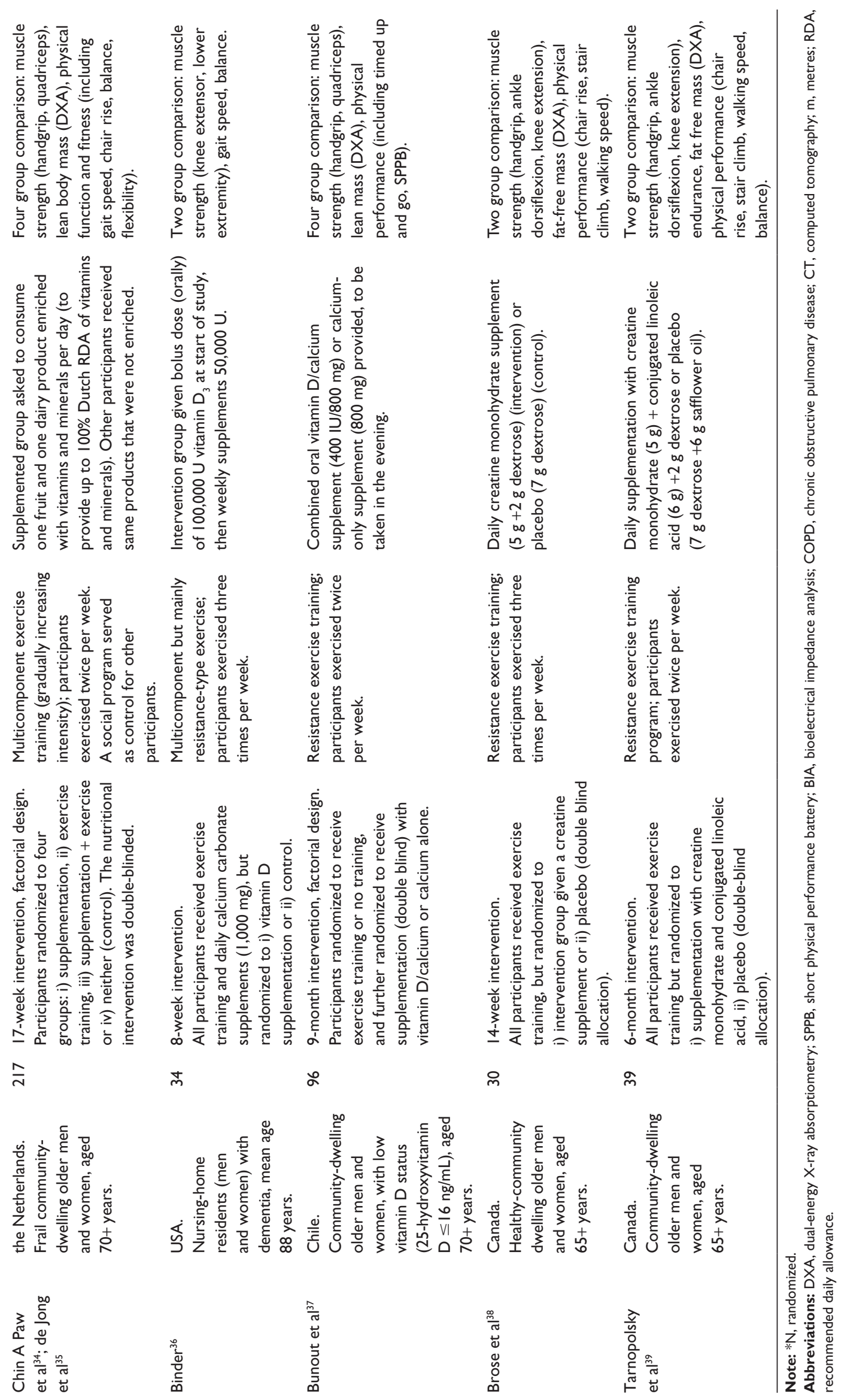
of a government nutrition program. ${ }^{32,33}$ Five of the six studies provided measures of muscle strength, size, and physical performance ${ }^{27-29,31-35}$ one provided measure of strength and physical performance, ${ }^{30}$ one provided a measure of muscle power. ${ }^{27}$

\section{Muscle strength}

The effects of exercise training on muscle strength differed across studies. There were clear improvements in some studies, ${ }^{28,29,32,33}$ smaller changes ${ }^{34,35}$ or no benefits ${ }^{27,30,31}$ described in others. With the exception of one study of older men and women with COPD, in which quadriceps muscle force increased significantly over the intervention period in the combined exercise/supplement group, additive or interactive effects of multinutrient supplementation and exercise training on muscle strength were not described.

\section{Muscle size}

The effects of exercise training on measures of muscle size differed across the five studies with measured outcomes, with reported increases in cross-sectional thigh muscle area and lean mass in two studies ${ }^{29,34}$ and no changes in fat free mass in others. ${ }^{27,31-33}$ A consistent finding was that there was no evidence of interactive effects of multinutrient supplementation with exercise training on measures of muscle size.

\section{Physical performance}

Exercise training was reported to have a positive effect on some or all of the physical performance measures in most studies, ${ }^{27-29,32-35}$ but there was no evidence of additive or interactive effects of multinutrient supplementation in the participants who received exercise training. However, among older participants with COPD, there was a significant improvement in exercise performance (measured as distance walked in 6 minutes) among participants who had received the multinutrient supplement together with exercise training. ${ }^{31}$

\section{Vitamin D supplementation}

Two of the 17 studies examined the combined effects of vitamin D supplementation and exercise training on muscle outcomes. One study included nursing home residents who had dementia, who were first given a bolus dose of vitamin D (100,000 U), followed by weekly supplements (50,000 U) over a period of 8 weeks. ${ }^{36}$ In the other, larger, study Bunout et $\mathrm{al}^{37}$ evaluated the effects of daily supplementation with $400 \mathrm{IU}$ over a period of 9 months in community-dwelling older adults who had low vitamin D status $(<16 \mathrm{ng} / \mathrm{mL}$ 25-hydroxyvitamin D). Both studies measured strength and assessed physical performance outcomes; in the second study body composition was assessed by dual-energy X-ray absorptiometry. ${ }^{37}$ Vitamin D status increased in the supplemented participants in both studies.

\section{Muscle strength}

Measured muscle strength increased following exercise training in both studies, although in the study of nursing home residents, analysis of the pooled results showed a decline in one measure of strength (knee extension). Neither study provided evidence of greater effects of exercise training on muscle strength among supplemented participants.

\section{Muscle size}

In the study that assessed body composition, there were no significant changes in any of the four comparison groups following the period of supplementation and exercise training. ${ }^{37}$

\section{Physical performance}

Exercise training significantly improved balance, but had no effect on gait speed in the study of nursing home residents; there were no differences in physical performance between supplemented and non-supplemented participants. ${ }^{36}$ In older adults with low vitamin D status, there was a significant improvement in the short physical performance battery among adults who had received exercise training. However, measured timed up and go improved more among trained subjects who had been supplemented with vitamin D. ${ }^{37}$

\section{Creatine supplementation}

Two studies examined the effects of creatine supplementation combined with exercise training on muscle outcomes. Participants in both studies were older Canadian adults who were community-dwelling, but the studies differed in duration (14 weeks ${ }^{38}$ versus 6 months). ${ }^{39}$ The dose of creatine monohydrate included in the daily supplements was the same $(5 \mathrm{~g})$ in both studies, but in the 6-month supplementation study the supplement also contained conjugated linoleic acid (6 g). ${ }^{39}$ Both studies measured muscle strength, size, and physical performance as outcomes; the 6-month study also included a measure of muscle endurance (number of repetitions completed). ${ }^{39}$

\section{Muscle strength}

In both studies, muscle strength increased following exercise training. There were some differences in the combined effects of creatine supplementation and exercise training in terms of outcomes and according to sex, when comparing studies, but overall a consistent finding was of greater improvements in strength in participants supplemented with 
creatine when compared with the placebo groups. ${ }^{38,39}$ For example, a 6-month period of creatine/conjugated linoleic acid supplementation did not further enhance isometric strength measurements after exercise training, but isokinetic knee extension strength was greater in both men and women who were supplemented. However, in the shorter study of creatine supplementation, men and women who were supplemented showed greater increases in isometric knee extension strength, and in men only, greater gains in isometric dorsiflexion strength. ${ }^{38}$ In the study in which muscle endurance was assessed, it increased significantly following exercise training, and greater improvements were observed in chest press and arm flexion among supplemented participants. ${ }^{39}$

\section{Muscle size}

There were inconsistent changes in fat free mass resulting from exercise training between the two studies. However, a consistent finding was that in both studies greater gains in fat free mass were found among supplemented participants who received exercise training, when compared to the placebo groups.

\section{Physical performance}

The findings of both studies were consistent, such that measured physical performance improved with exercise training, but there was no evidence of additional benefits arising from supplementation.

\section{Discussion}

In a comprehensive review of the literature, we considered 17 studies of older adults ( $\geq 65$ years) in which combined nutrition and exercise interventions were used to increase muscle strength and/or mass, and achieve improvements in physical performance. The studies were diverse in terms of the participants included (nutritional status and degree of physical frailty), supplementation strategies (differences in nutrients and doses), exercise training (type and frequency), as well as design (duration and setting). This diversity has implications for the review and the extent to which data can be collated. The main finding is that enhanced benefits of exercise training, when combined with dietary supplementation, have been shown in some trials, indicating its potential as an intervention strategy, but that existing evidence is inconsistent. Its overall message is that there are many gaps in the evidence base and to address these, further studies are needed.

Seven studies included in this review evaluated the effects of provision of additional amino acids or protein in combination with exercise training. The supplements differed in type, dose, frequency, and delivery (provided at the same time as exercise or not) across studies, and participants ranged in status from healthy older adults to those who were frail or sarcopenic. In only three studies was there evidence of additional gains resulting from protein or amino acid supplementation combined with exercise training, in muscle strength $^{25}$ or lean mass. ${ }^{22,26}$ There is considerable interest in dietary protein requirements in older age, and whether these should be raised to counteract effects of anabolic resistance. However, insufficient habitual protein intakes may not be very common ${ }^{40}$ - and it is possible that enhanced effects of exercise training arising from protein supplementation would only be expected in the sub-group of the population who have low intakes. Adequate protein intake at baseline was highlighted as a possible explanation for the lack of interactive effects in three of the studies included in this review. ${ }^{19,23,24}$ Another explanation may be that there are differing effects according to source or quality of protein. ${ }^{41} \mathrm{In}$ two recent trials that were excluded from this review (because of age of participants and/or publication date), evidence of interactive effects of meat supplementation, ${ }^{41}$ but not milk supplementation, ${ }^{42}$ on muscle mass and strength have been shown. Further studies are needed to establish the role of type of protein, and whether there are important additional benefits for vulnerable older adults in the population whose habitual protein intakes are low.

The combined effects of supplementation with a range of nutrients together with exercise training were evaluated in six studies included in this review. There was diversity across studies in terms of the composition of supplements used, but with one exception in which fortified foods were provided to increase micronutrient intake only in the intervention groups, ${ }^{34,35}$ they contained a mixture of micro- and macronutrients. One of the studies included in the review provided evidence of enhanced benefits of exercise training when combined with multinutrient supplementation. In the remaining five studies, no such enhanced effects were seen. In view of a growing evidence base in which older adults who have "healthier" diets, providing higher intakes of a range of micronutrients, have better physical function, ${ }^{13,40}$ these negative findings may be surprising. One possibility is that baseline nutritional status of participants in the included studies was not sufficiently compromised to see effects of supplementation. ${ }^{35}$ However, a second is that healthier diets provide greater intakes of a range of other micronutrients and bioactive non-nutrients ${ }^{43}$ that were not included in the supplements used in the trials. Examples include phytochemicals such as flavonoids and long-chain polyunsaturated fatty acids, that have anti-inflammatory actions, that may have important effects on muscle function. Consistent with this 
possibility, in a recent study of strength training in a group of older women (mean age 64 years), fish oil supplementation resulted in greater improvements in muscle strength and functional capacity. ${ }^{44}$ It may also be noteworthy that the study included in the present review that showed enhanced effects of exercise training with multinutrient supplementation, provided participants with $4 \mathrm{~g}$ long-chain polyunsaturated fatty acids per day in addition to a range of macro- and micronutrients. ${ }^{31}$ Dietary modification may be a more effective way of increasing intakes of a wide range of nutrients and non-nutrients than using dietary supplements. But further studies are needed to address the effects of supplementation as well as interventions to change dietary patterns, on muscle strength and physical performance of older adults.

Two studies included in the review evaluated the interactive effects of vitamin D supplementation and exercise training. One study included elderly dementia patients who were provided with weekly supplements over an 8-week period; the other study included community-dwelling adults who had low vitamin D status and who were provided with daily supplements for a period of 9 months. No additive effects were shown in most outcomes, even among vitamin D-deficient adults. However, there were differences in the timed up and go assessment, such that this measure of physical performance improved more in the trained participants supplemented with vitamin D. ${ }^{37}$ Further studies are needed.

There were two studies that met our search criteria that evaluated the combined effects of exercise training and creatine supplementation; the studies were similar in terms of population studied and the daily dose of supplement, but differed in duration. ${ }^{38,39}$ Although there were some differences in outcomes and between sexes, both studies found enhanced benefits of exercise training in the supplemented participants, such that measures of strength and fat free mass were greater, when compared with the control groups. However, in both cases, these differences did not translate into improvements in physical performance within the period of study. Although more data are needed, these findings are consistent with the recent meta-analysis of data from 357 older adults, in which enhanced benefits of exercise training were found when combined with creatine supplementation. ${ }^{45}$ However, whilst these findings are encouraging the number of studies is small and further work is needed. ${ }^{45}$

\section{Conclusion}

We reviewed 17 intervention studies that used a combination of exercise training and nutritional supplementation to improve muscle outcomes in older people. The finding of enhanced benefits of exercise training when combined with dietary supplementation in some trials, highlights its potential as a strategy for the prevention and management of sarcopenia. However, existing evidence is based on populations who differ in age, frailty, and nutritional status - and findings are inconsistent. The many gaps in current understanding mean there is insufficient evidence on which to base recommendations. ${ }^{4}$ Further studies, particularly that evaluate the effects of exercise training combined with dietary strategies to increase intakes of a range of nutrients as well as bioactive non-nutrients, are needed.

\section{Disclosure}

None of the authors have any conflict of interest in relation to this paper.

\section{References}

1. Sayer AA. Sarcopenia. BMJ. 2010;341:c4097.

2. Patel HP, Syddall HE, Jameson K, et al. Prevalence of sarcopenia in community-dwelling older people in the UK using the European Working Group on Sarcopenia in Older People (EWGSOP) definition: findings from the Hertfordshire Cohort Study (HCS). Age Ageing. 2013; 42(3):378-384.

3. Masanes F, Culla A, Navarro-Gonzalez M, et al. Prevalence of sarcopenia in healthy community-dwelling elderly in an urban area of Barcelona (Spain). J Nutr Health Aging. 2012;16(2):184-187.

4. Cruz-Jentoft AJ, Landi F, Schneider SM, et al. Prevalence of and interventions for sarcopenia in ageing adults: a systematic review. Report of the International Sarcopenia Initiative (EWGSOP and IWGS). Age Ageing. 2014;43(6):748-759.

5. Sayer AA, Dennison EM, Syddall HE, Gilbody HJ, Phillips DI, Cooper C. Type 2 diabetes, muscle strength, and impaired physical function: the tip of the iceberg? Diabetes Care. 2005;28(10):2541-2542.

6. Morley JE, Anker SD, von Haehling S. Prevalence, incidence, and clinical impact of sarcopenia: facts, numbers, and epidemiology-update 2014. J Cachexia Sarcopenia Muscle. 2014;5(4):253-259.

7. Batsis JA, Mackenzie TA, Barre LK, Lopez-Jimenez F, Bartels SJ. Sarcopenia, sarcopenic obesity and mortality in older adults: results from the National Health and Nutrition Examination Survey III. Eur $J$ Clin Nutr. 2014;68(9):1001-1007.

8. Cooper R, Kuh D, Hardy R. Objectively measured physical capability levels and mortality: systematic review and meta-analysis. $B M J$. 2010;341:c4467.

9. Janssen I, Shepard DS, Katzmarzyk PT, Roubenoff R. The healthcare costs of sarcopenia in the United States. J Am Geriatr Soc. 2004; 52(1):80-85.

10. Cruz-Jentoft AJ, Baeyens JP, Bauer JM, et al. Sarcopenia: European consensus on definition and diagnosis: Report of the European Working Group on Sarcopenia in Older People. Age Ageing. 2010;39(4): 412-423.

11. Fielding RA, Vellas B, Evans WJ, et al. Sarcopenia: an undiagnosed condition in older adults. Current consensus definition: prevalence, etiology, and consequences. International working group on sarcopenia. J Am Med Dir Assoc. 2011;12(4):249-256.

12. Montero-Fernández N1, Serra-Rexach JA. Role of exercise on sarcopenia in the elderly. Eur J Phys Rehabil Med. 2013;49(1):131-143.

13. Robinson S, Cooper C, Aihie Sayer A. Nutrition and sarcopenia: a review of the evidence and implications for preventive strategies. J Aging Res. 2012;2012:510801. 
14. SayerAA, Robinson SM, PatelHP, Shavlakadze T, CooperC, GroundsMD. New horizons in the pathogenesis, diagnosis and management of sarcopenia. Age Ageing. 2013;42(2):145-150.

15. Liu CJ, Latham NK. Progressive resistance strength training for improving physical function in older adults. Cochrane Database Syst Rev. 2009;(3): CD002759.

16. Cermak NM, Res PT, de Groot LC, Saris WH, van Loon LJ. Protein supplementation augments the adaptive response of skeletal muscle to resistance-type exercise training: a meta-analysis. Am J Clin Nutr. 2012 96(6):1454-1464.

17. Daly RM, Duckham RL, Gianoudis J. Evidence for an interaction between exercise and nutrition for improving bone and muscle health. Curr Osteoporos Rep. 2014;12(2):219-226.

18. Candow DG, Forbes SC, Little JP, Cornish SM, Pinkoski C, Chilibeck PD Effect of nutritional interventions and resistance exercise on aging muscle mass and strength. Biogerontology. 2012;13(4):345-358.

19. Arnarson A, Gudny Geirsdottir O, Ramel A, Briem K, Jonsson PV, Thorsdottir I. Effects of whey proteins and carbohydrates on the efficacy of resistance training in elderly people: double blind, randomised controlled trial. Eur J Clin Nutr. 2013;67(8):821-826.

20. Rosendahl E, Lindelöf N, Littbrand H, et al. High-intensity functional exercise program and protein-enriched energy supplement for older persons dependent in activities of daily living: a randomised controlled trial. Aust J Physiother. 2006;52(2):105-113.

21. Carlsson M, Littbrand H, Gustafson Y, et al. Effects of high-intensity exercise and protein supplement on muscle mass in ADL dependent older people with and without malnutrition: a randomized controlled trial. J Nutr Health Aging. 2011;15(7):554-560.

22. Tieland M, van de Rest O, Dirks ML, et al. Protein supplementation improves physical performance in frail elderly people: a randomized, double-blind, placebo-controlled trial. J Am Med Dir Assoc. 2012; 13(8):720-726.

23. Verdijk LB, Jonkers RA, Gleeson BG, et al. Protein supplementation before and after exercise does not further augment skeletal muscle hypertrophy after resistance training in elderly men. Am J Clin Nutr. 2009; 89(2):608-616.

24. Godard MP, Williamson DL, Trappe SW. Oral amino-acid provision does not affect muscle strength or size gains in older men. Med Sci Sports Exerc. 2002;34(7):1126-1131.

25. Kim HK, Suzuki T, Saito K, et al. Effects of exercise and amino acid supplementation on body composition and physical function in community-dwelling elderly Japanese sarcopenic women: a randomized controlled trial. J Am Geriatr Soc. 2012;60(1):16-23.

26. Vukovich MD, Stubbs NB, Bohlken RM. Body composition in 70-yearold adults responds to dietary beta-hydroxy-beta-methylbutyrate similarly to that of young adults. J Nutr. 2001;131(7):2049-2052.

27. Bonnefoy M, Cornu C, Normand S, et al. The effects of exercise and protein-energy supplements on body composition and muscle function in frail elderly individuals: a long-term controlled randomised study. Br J Nutr. 2003;89(5):731-739.

28. Fiatarone MA, O'Neill EF, Ryan ND, et al. Exercise training and nutritional supplementation for physical frailty in very elderly people. N Engl J Med. 1994;330(25):1769-1775.

29. Fiatarone MA, O’Neill EF, Doyle N, et al. The Boston FICSIT study: the effects of resistance training and nutritional supplementation on physical frailty in the oldest old. J Am Geriatr Soc. 1993;41(3):333-337.
30. Miller MD, Crotty M, Whitehead C, Bannerman E, Daniels LA. Nutritional supplementation and resistance training in nutritionally at risk older adults following lower limb fracture: a randomized controlled trial. Clin Rehabil. 2006;20(4):311-323.

31. Sugawara K, Takahashi H, Kashiwagura T, et al. Effect of antiinflammatory supplementation with whey peptide and exercise therapy in patients with COPD. Respir Med. 2012;106(11):1526-1534.

32. Bunout D, Barrera G, de la Maza P, et al. The impact of nutritional supplementation and resistance training on the health functioning of freeliving Chilean elders: results of 18 months of follow-up. J Nutr. 2001; 131(9):2441S-2446S.

33. Bunout D, Barrera G, de la Maza P, et al. Effects of nutritional supplementation and resistance training on muscle strength in free living elders. Results of one year follow. J Nutr Health Aging. 2004;8(2):68-75.

34. Chin A Paw MJ, de Jong N, Schouten EG, Hiddink GJ, Kok FJ. Physical exercise and/or enriched foods for functional improvement in frail, independently living elderly: a randomized controlled trial. Arch Phys Med Rehabil. 2001;82(6):811-817.

35. de Jong N, Chin A Paw MJ, de Groot LC, Hiddink GJ, van Staveren WA. Dietary supplements and physical exercise affecting bone and body composition in frail elderly persons. Am J Public Health. 2000;90(6): 947-954.

36. Binder EF. Implementing a Structured Exercise Program for Frail Nursing Home Residents With Dementia: Issues and Challenges. J Aging Phys Act. 1995;3:383-395.

37. Bunout D, Barrera G, Leiva L, et al. Effects of vitamin D supplementation and exercise training on physical performance in Chilean vitamin D deficient elderly subjects. Exp Gerontol. 2006;41(8):746-752.

38. Brose A, Parise G, Tarnopolsky MA. Creatine supplementation enhances isometric strength and body composition improvements following strength exercise training in older adults. J Gerontol A Biol Sci Med Sci. 2003;58(1):11-19.

39. Tarnopolsky M, Zimmer A, Paikin J, et al. Creatine monohydrate and conjugated linoleic acid improve strength and body composition following resistance exercise in older adults. PLoS One. 2007;2(10):e991.

40. Millward DJ. Nutrition and sarcopenia: evidence for an interaction. Proc Nutr Soc. 2012;71(4):566-575.

41. Daly RM, O'Connell SL, Mundell NL, Grimes CA, Dunstan DW, Nowson CA. Protein-enriched diet, with the use of lean red meat, combined with progressive resistance training enhances lean tissue mass and muscle strength and reduces circulating IL- 6 concentrations in elderly women: a cluster randomized controlled trial. Am J Clin Nutr. 2014; 99(4):899-910.

42. Kukuljan S, Nowson CA, Sanders K, Daly RM. Effects of resistance exercise and fortified milk on skeletal muscle mass, muscle size, and functional performance in middle-aged and older men: an 18-mo randomized controlled trial. J Appl Physiol (1985). 2009;107(6):1864-1873.

43. Mathers JC. Impact of nutrition on the ageing process. Br J Nutr. 2015; 113 Suppl:S18-S22.

44. Rodacki CL, Rodacki AL, Pereira G, et al. Fish-oil supplementation enhances the effects of strength training in elderly women. Am J Clin Nutr. 2012;95(2):428-436.

45. Devries MC, Phillips SM. Creatine supplementation during resistance training in older adults-a meta-analysis. Med Sci Sports Exerc. 2014; 46(6):1194-203
Clinical Interventions in Aging

\section{Publish your work in this journal}

Clinical Interventions in Aging is an international, peer-reviewed journal focusing on evidence-based reports on the value or lack thereof of treatments intended to prevent or delay the onset of maladaptive correlates of aging in human beings. This journal is indexed on PubMed Central, MedLine,

\section{Dovepress}

CAS, Scopus and the Elsevier Bibliographic databases. The manuscript management system is completely online and includes a very quick and fair peer-review system, which is all easy to use. Visit http://www.dovepress. com/testimonials.php to read real quotes from published authors. 